\title{
A Low Phase Noise, Low Power and Wide Tuning Range VCO with Filtering Technique in ISM Band
}

\author{
Masoud Sabaghi ${ }^{*}$, Saeid Marjani' ${ }^{2}$, Abbas Majdabadi1 \\ ${ }^{1}$ Laser and Optics Research School, Nuclear Science and Technology Research School, \\ Atomic Energy Organization of Iran, Tehran, Iran \\ ${ }^{2}$ Department of Electrical Engineering, Ferdowsi University of Mashhad, Mashhad, Iran \\ Email: *msabaghi@aeoi.org.ir
}

Received 8 January 2016; accepted 14 February 2016; published 17 February 2016

Copyright (C) 2016 by authors and Scientific Research Publishing Inc.

This work is licensed under the Creative Commons Attribution International License (CC BY).

http://creativecommons.org/licenses/by/4.0/

(c) (i) Open Access

\begin{abstract}
In this paper, a novel voltage controlled oscillator (VCO) with low phase noise, low power consumption and wide tuning range in the industrial, scientific and medical (ISM) band is proposed for communication systems applications. For improving the phase noise, filtering technique is used and VCO is designed with TSMC CMOS $0.18 \mu \mathrm{m}$ technology and the power supply is $1.5 \mathrm{~V}$. The simulation results with advanced design system (ADS) shows that phase noise in 1 MHz offset frequency from the carrier is $-122 \mathrm{dBc} / \mathrm{Hz}$ and tuning range is 2 to $2.8 \mathrm{GHz}$. The power consumption of the core is $2.49 \mathrm{~mW}$.
\end{abstract}

\section{Keywords}

Filtering Technique, Tuning Range, Phase Noise, Power Consumption, Voltage Controlled Oscillators (VCO)

\section{Introduction}

One of the most important parts in transmitters is phase locked loop (PLL), and their performance is strongly influenced by the voltage controlled oscillators (VCO) [1]. VCO is one of the newest comparator types [2]-[4]. VCO-based comparator and quantizer are one of the most important sections of successive approximation ADC and delta-sigma, respectively [5]-[10]. The similarity between electrical oscillators and optical oscillators including lasers [11]-[20] and vertical-cavity surface-emitting lasers (VCSELs) [21]-[33] allows the processes

${ }^{*}$ Corresponding author.

How to cite this paper: Sabaghi, M., Marjani, S. and Majdabadi, A. (2016) A Low Phase Noise, Low Power and Wide Tuning Range VCO with Filtering Technique in ISM Band. Circuits and Systems, 7, 51-57.

http://dx.doi.org/10.4236/cs.2016.72006 
used in optical spectroscopy to be applied. VCO is one of the most difficult circuits to integrate for the following reasons: 1) poor quality factor of the monolithic inductor; 2) limited tuning range of the varactor; and 3) poor flicker noise in CMOS technology compared with the other technologies such as SiGe HBT [34]. There are many methods to improve the phase noise, an example is the multigated transistor which is proposed in [35].

The important goals in designing of VCO are low power dissipation, low phase noise and wide tuning range. In this paper, with using the filtering technique and good selecting of the inductor and the varactor, a LC-VCO with low power $(2.49 \mathrm{~mW})$, low phase noise $(-122 \mathrm{dBc} / \mathrm{Hz})$ and wide band is proposed. The rest of this paper is as follows. In Section 2, the VCO design considering the conventional cross-coupled architecture is reviewed and the proposed VCO is introduced in order to reach low power consumption, low phase noise and wide tuning range. Section 3 presents the results and discussions. Finally, we conclude in Section 4.

\section{Proposed Voltage-Controlled Oscillator}

There are different structures for designing oscillators. As an example Ring oscillator and LC Tank structures can be noted. LC tank oscillators can be further divided to Colpits, Hartely and cross coupled oscillators. In this paper cross coupled structure which has a low phase noise is used This cross coupled structure is also suitable for integration. Cross coupled oscillators are one of the most commonly used structures [36]. In Figure 1 the conventional cross coupled oscillator is shown.

The LC tank forms the most important part of the cross coupled oscillators. The integrated circuits oscillators like the other blocks of the transmitter, suffer from the low quality factor of the integrated inductors. Therefore a versatile design of the LC tank is very important. The resonator of the VCO consists of MOS varactors for continuous tuning and an on-chip inductor. MOS varactors $\left(\mathrm{C}_{\mathrm{var}}\right)$ are employed to provide the frequency tuning capability. The capacitance of MOS varactors determines the oscillation frequency together with the inductance and other parasitic capacitances [37].

As power supply, PMOS current source is generally used because the PMOS transistors have less noise flicker [38]. Therefore in the proposed VCO this type of current source is used. Figure 2 shows the PMOS current source that is used in the proposed circuit.

The most important parameter in a VCO is phase noise. To calculate the phase noise value, different relationships is presented [34]. The most famous of these relations is Leeson equation [39]. Leeson's model defines the phase noise at a given offset frequency, $\Delta \omega$, from the center frequency as bellow:

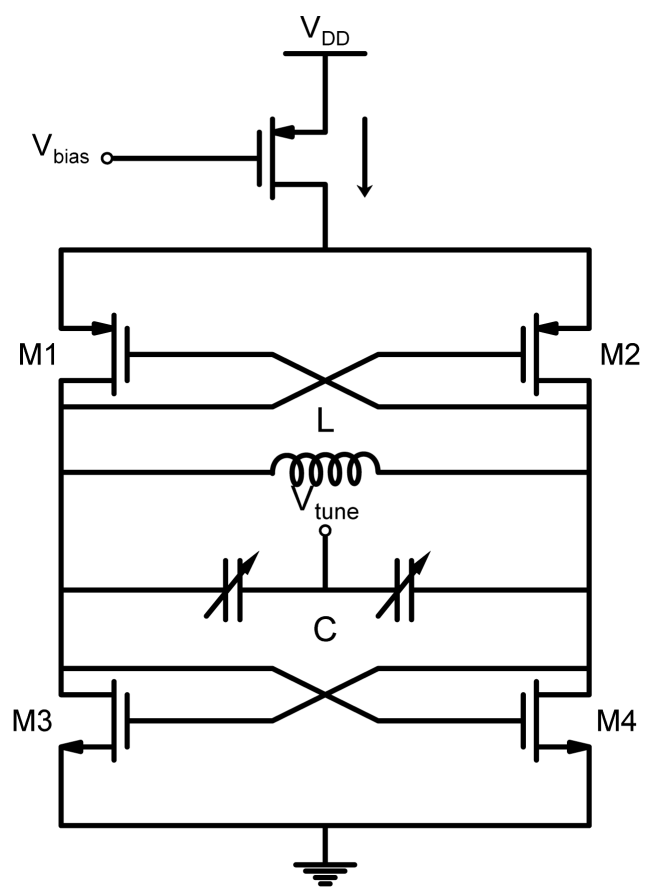

Figure 1. CMOS complementary voltage-controlled oscillator [36]. 


$$
L[\Delta \omega]=10 \log \left[\frac{2 F K T}{P_{\text {sig }}}\left[1+\left(\frac{\omega_{0}}{2 Q \Delta \omega}\right)^{2}\right]\left(1+\frac{\Delta \omega_{1 / f^{3}}}{|\Delta \omega|}\right)\right]
$$

where $\mathrm{L}[\Delta \omega]$ is the phase noise at offset frequency $\Delta \omega$ from the operating frequency $\omega_{0}, \mathrm{~F}$ is an empirical fitting factor, and $\mathrm{Q}$ is quality factor of the LC-tank. $\omega_{0}$ is the oscillation frequency as bellow:

$$
\omega_{0}=\frac{1}{\sqrt{L C}} \text {. }
$$

Current source noise creates phase noise in the oscillator [40]. The filtering technique is an effective method for improving the phase noise. In this technique the second harmonic noises in the current source which are efficient in creating phase noise is blocked [38]. Figure 3 shows the proposed VCO. In this scheme a high value capacitor is connected in parallel with the current source and shunts the second harmonic noises of the current to the ground. To further reduce the amplitude of the second harmonic of the current, an impedance boosting inductor is included at the common drain node in the design. The inductance $\left(\mathrm{L}_{2}\right)$, is selected for resonating at $2 \omega_{0}$ with the total capacitance in the common mode.

\section{Simulation Results}

The proposed VCO is simulated by Advanced Design System (ADS) in TSMC $0.18 \mu$ CMOS process. The tuning range of the $\mathrm{VCO}$ is from 2.04 to $2.79 \mathrm{GHz}$ that is achieved by the tuning voltage from 0 to $1.5 \mathrm{~V}$, as shown in Figure 4. Figure 5 shows that the proposed VCO at $2.7 \mathrm{GHz}$ central frequency has the phase noise of $-122 \mathrm{dBc} / \mathrm{Hz}$ at $1 \mathrm{MHz}$ offset. Table 1 provides comparison of the proposed VCO and the most recently works. As seen, its phase noise is small compared to previous results. Figure 6 shows the output power of the VCO versus tuning voltage, after connection to the output buffer. The power consumption of the circuit is $2.49 \mathrm{~mW}$.

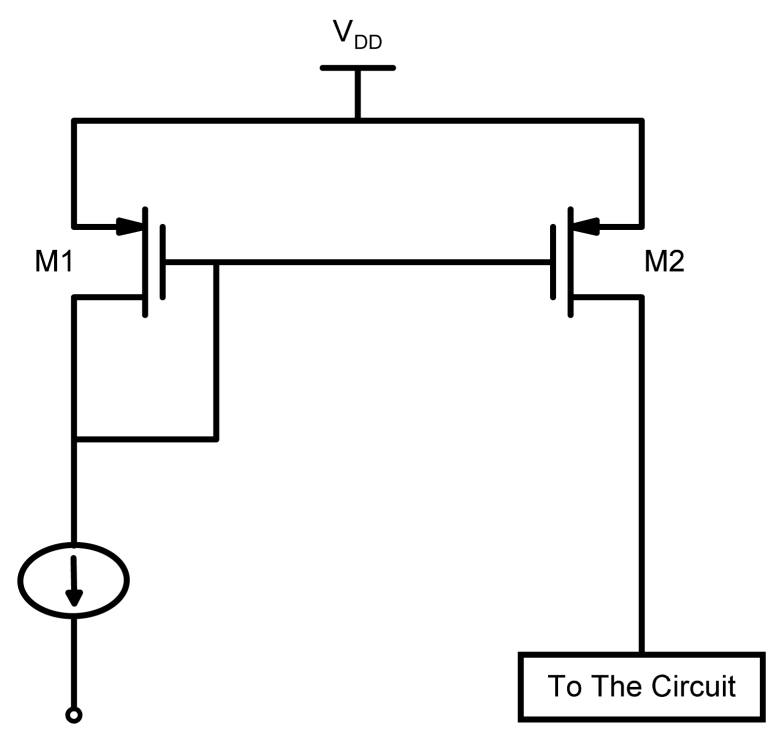

Figure 2. PMOS current source.

Table 1. Specification comparison of the proposed VCO and the other works.

\begin{tabular}{cccc}
\hline specification & This Work & [35] [42] & [41] \\
\hline Frequency (GHz) & $2.04-2.79$ & $2.17-2.7$ & 2.4 \\
Phase Noise & $122 @ 1 \mathrm{MHz}$ & $122 @ 1 \mathrm{MHz}$ & $131 @ 3 \mathrm{MHz}$ \\
Power Consumption & $2.49 \mathrm{~mW}$ & $2.7 \mathrm{~mW}$ & $135 @ 3 \mathrm{MHz}$ \\
Power Supply & $1.5 \mathrm{~V}$ & $0.9 \mathrm{~V}$ & $1.8 \mathrm{~V}$ \\
\hline
\end{tabular}




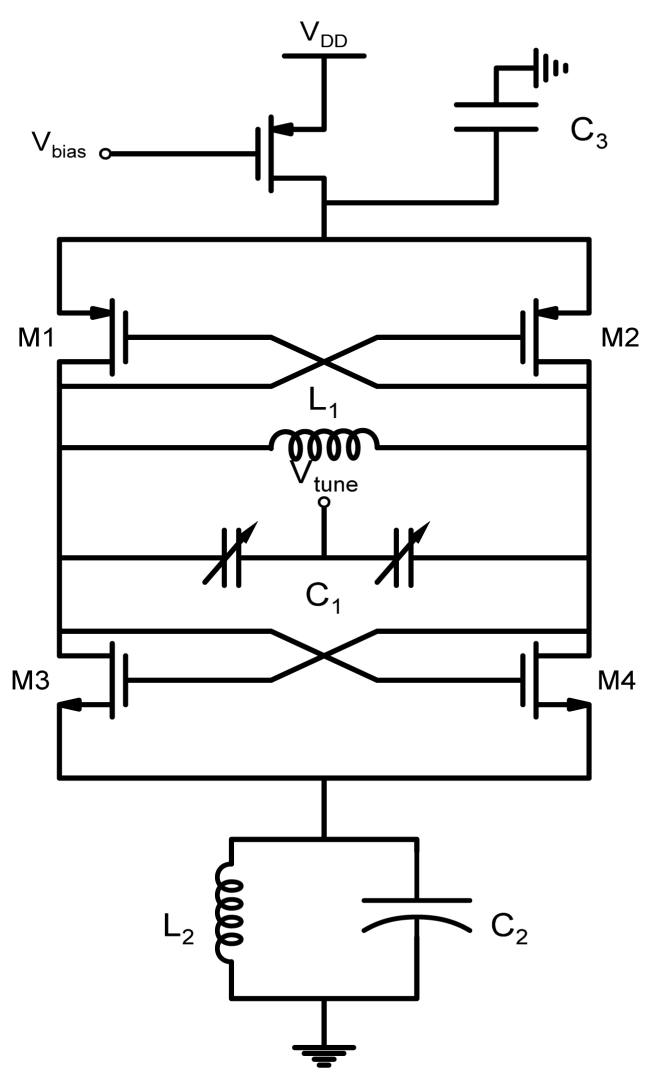

Figure 3. Proposed VCO scheme with the PMOS current source.

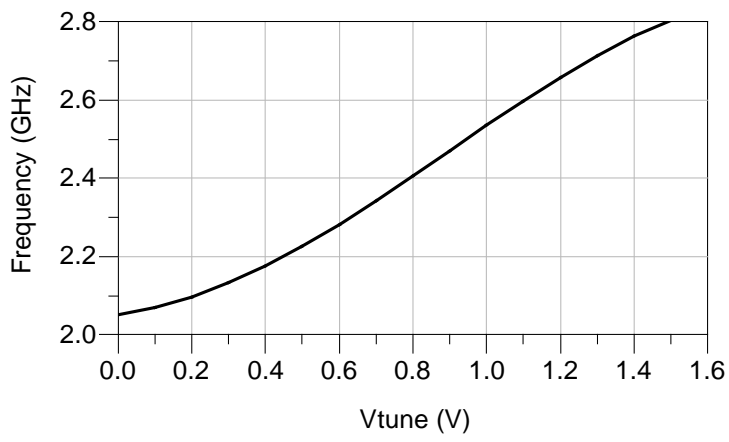

Figure 4. Oscillation frequency versus control voltage.

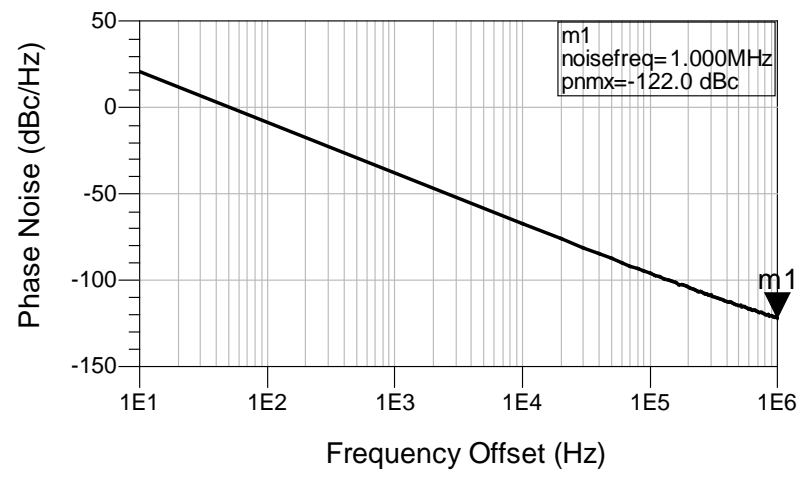

Figure 5. Phase noise of the VCO in the $1 \mathrm{MHz}$ offset. 


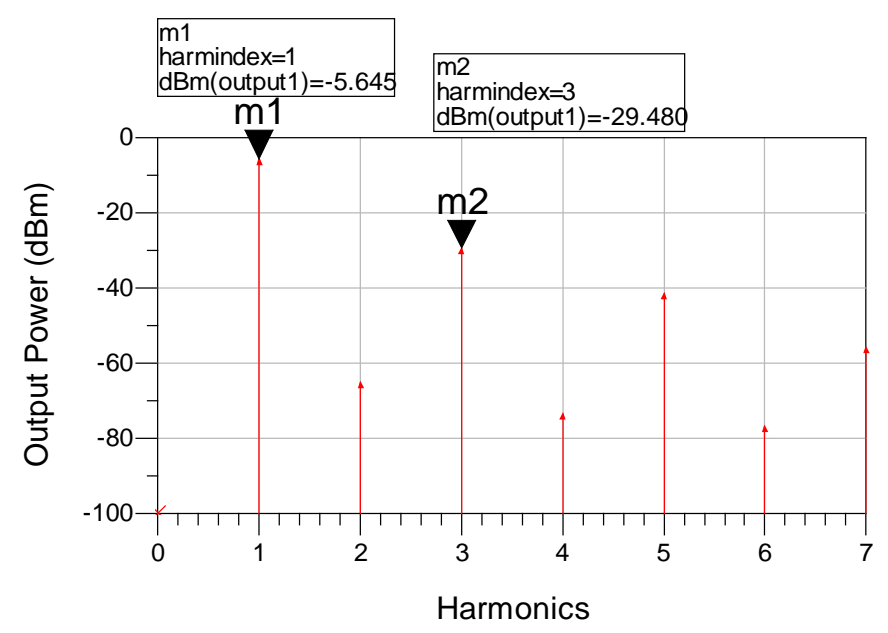

Figure 6. Output power of the VCO.

\section{Conclusion}

A novel voltage controlled oscillator design using filtering technique is presented. The simulation results show that the proposed VCO at $2.7 \mathrm{GHz}$ central frequency has the phase noise of $-122 \mathrm{dBc} \backslash \mathrm{Hz}$ at $1 \mathrm{MHz}$ offset. The power consumption of the VCO core is $2.49 \mathrm{~mW}$ and the tuning range is 2.04 to $2.8 \mathrm{GHz}$ with 0 to $1.5 \mathrm{~V}$ voltage control.

\section{Acknowledgements}

This work was supported by the Laser and Optics Research School, Nuclear Science and Technology Research School, Atomic Energy Organization of Iran, Tehran, Iran.

\section{References}

[1] Volkovskii, A.R., Tsimring, L.Sh., Rulkov, N.F. and Langmore, I. (2005) Spread Spectrum Communication System with Chaotic Frequency Modulation. Chaos, 15, 1-6. http://dx.doi.org/10.1063/1.1942327

[2] Dashtbayazi, M., Sabaghi, M. and Marjani, S. (2015) Dynamic Comparator with Using Negative Resistance and CMOS Input Pair Strategies in FS $=4 \mathrm{MHz}-10 \mathrm{GHz}$. Journal of Electrical and Electronic Engineering, 3, 93-96. http://dx.doi.org/10.11648/j.jeee.20150304.15

[3] Dashtbayazi, M., Marjani, S. and Sabaghi, M. (2015) A 4 MHz - 10 GHz, 10-ps/dec Dynamic Comparator Using Negative Resistance Combined with CMOS Input Pair. The Progress in Electromagnetics Research Symposium (PIERS), Prague, Czech Republic, 6-9 July 2015, 831-834.

[4] Sabaghi, M., Majdabadi, A., Dashtbayazi, M. and Marjani, S. (2015) A 4 MHz - 10 GHz, Dynamic Comparator Using Negative Resistance Combined with CMOS Input Pair Strategy in Dynamic Pre-Amplifier. The Iranian Conference on Optics and Laser Engineering (ICOLE), Isfahan, Iran, 2-3 September 2015, 136-139.

[5] Dashtbayazi, M., Sabaghi, M. and Marjani, S. (2015) An Optimized DAC Timing Strategy in SAR ADC with Considering the Overshoot Effect. Journal of Electrical and Electronic Engineering, 3, 19-24. http://dx.doi.org/10.11648/j.jeee.20150302.12

[6] Dashtbayazi, M., Sabaghi, M., Rezaei, M. and Marjani, S. (2014) New Delta Sigma Modulator Structure Using Second Order Filter in One Stage Technique. Journal of Electrical and Electronic Engineering, 2, 82-88. http://dx.doi.org/10.11648/j.jeee.20140206.11

[7] Sabaghi, M., Dashtbayazi, M. and Marjani, S. (2016) Dynamic Hysteresis Band Fixed Frequency Current Control. World Applied Programming, 6, 1-4.

[8] Sabaghi, M., Dashtbayazi, M. and Marjani, S. (2015) An Optimized Opamp-Sharing in 2nd Order $\Delta \Sigma$ Modulator Based on Changing the Stages Output Capacitance Timing Strategy. The Progress in Electromagnetics Research Symposium (PIERS), Prague, Czech Republic, 6-9 July 2015, 827-830.

[9] Dashtbayazi, M., Majdabadi, A., Sabaghi, M. and Marjani, S. (2015) An Optimized Opamp-Sharing Technique in 2nd Order Delta-Sigma Modulator Based on Changing the Stages Output Capacitance Timing Strategy. The Iranian Con- 
ference on Optics and Laser Engineering (ICOLE), Isfahan, Iran, 2-3 September 2015, 129-131.

[10] Dashtbayazi, M., Marjani, S., Sabaghi, M. and Majdabadi, A. Changing the Clock Pulse-Width Dedicated to Stages Strategy for Opamp-Sharing Technique in 2nd Order High-Pass Delta-Sigma Modulators. The International Conference on Electrical, Computer, Mechanical and Mechatronics Engineering (ICE), Dubai, United Arab Emirates, 4-5 February 2016, Unpublished.

[11] Marjani, A., Marjani, S. and Shirazian, S. (2011) Numerical Simulation of Silicon Carbide Polymers (6H-SiC \& 3C-SiC) as the Active Area for $0.83 \mu \mathrm{m}$ Wavelength Semiconductor Laser. The 14th Iranian Physical Chemistry Conference, Kish, 25-28 February 2011, 876-878.

[12] Marjani, S., Faez, R. and Marjani, H. (2011) Analysis and Design of Semiconductor Laser with Silicon Carbide Polymers (6H-SiC and 3C-SiC). Australian Journal of Basic and Applied Sciences, 5, 1060-1063.

[13] Marjani, S. and Marjani, H. (2012) Self-Heating Effects in a Silicon Carbide Polymers (6H-SiC and 3C-SiC) Semiconductor Laser. Asian Journal of Chemistry, 24, 3145-3147.

[14] Marjani, S. and Marjani, H. (2012) Effects of Lattice Temperature on the Various Elements of Heat Sources in Silicon Carbide Polymers (6H-SiC and 3C-SiC) Semiconductor Laser. Asian Journal of Chemistry, 24, 3123-3125.

[15] Marjani, S., Faez, R. and Marjani, H. (2012) Analysis of the Various Elements of Heat Sources in Silicon Carbide Polymers (6H-SiC and 3C-SiC) Semiconductor Laser. Asian Journal of Chemistry, 24, 2333-2335.

[16] Marjani, S., Faez, R. and Marjani, H. (2012) Design and Modeling of a Semiconductor Laser by Employing Silicon Carbide Polymers (6H-SiC, 3C-SiC and 4H-SiC). Asian Journal of Chemistry, 24, 2177-2179.

[17] Marjani, S., Faez, R. and Hosseini, S.E. (2013) Analysis of Lattice Temperature Effects on a GaInP/6H-SiC Strained Quantum-Well Lasers. Asian Journal of Chemistry, 25, 4715-4717.

[18] Madadi, R., Marjani, S. and Faez, R. (2013) Silicon Carbide Polymers (6H-SiC, 3C-SiC and 4H-SiC) Semiconductor Laser: Influence of Self-Heating. The 3rd Iranian Conference on Optics and Laser Engineering (ICOLE), Isfahan, 9-10 October 2013, 1069-1072.

[19] Rafighi, F., Behrouzinia, S., Khorasani, K., Sabaghi, M. and Marjani, S. (2016) The Electrical Parameters Modeling and Ex-Perimentation of Copper Vapor Laser. Circuits and Systems, 7, 23-28. http://dx.doi.org/10.4236/cs.2016.71003

[20] Behrouzinia, S., Khorasani, K., Marjani, S., Sabaghi, M., Aeinehvand, M.E. and Mohammadpour Lima, S. (2016) Experimental Study of Buffer Gas Flow Rate Effect on Output Power of a Copper Vapor Laser. Optics and Photonics Journal, in Press.

[21] Faez, R., Marjani, A. and Marjani, S. (2011) Design and Simulation of a High Power Single Mode 1550nm InGaAsP VCSELs. IEICE Electronics Express, 8, 1096-1101. http://dx.doi.org/10.1587/elex.8.1096

[22] Marjani, S., Faez, R. and Marjani, H. (2011) An Impact of the Hole Etching Depth within a Photonic Crystal VCSEL on Its Heat Sources. Australian Journal of Basic and Applied Sciences, 5, 766-770.

[23] Marjani, S., Faez, R. and Marjan, A. (2011) Design and Modeling of a High Single Mode Power Long Wavelength InGaAsP Photonic Crystal VCSEL. Australian Journal of Basic and Applied Sciences, 5, 1064-1069.

[24] Marjani, S., Rahnama, M. and Marjani, H. (2011) Numerical Optimization of Single-Mode InGaAsP Vertical-Cavity Surface-Emitting Lasers. Australian Journal of Basic and Applied Sciences, 5, 1207-1211.

[25] Marjani, S. and Marjani, H. (2011) Effects of Lattice Temperature on the Various Elements of Heat Sources in a Long Wavelength InGaAsP Photonic Crystal VCSEL. Australian Journal of Basic and Applied Sciences, 5, 1257-1261.

[26] Marjani, S. and Marjani, H. (2011) Analysis of Lattice Temperature Effects on a Long Wavelength InGaAsP Photonic Crystal VCSEL. Australian Journal of Basic and Applied Sciences, 5, 1374-1378.

[27] Marjani, S. and Marjani, H. (2012) Optimization of a Long Wavelength Vertical-Cavity Surface-Emitting Lasers by Employing Photonic Crystal. Asian Journal of Chemistry, 24, 3174-3176.

[28] Marjani, S. and Marjani, H. (2012) Effects of Hole Etching Depth in a Long Wavelength InGaAsP Photonic Crystal Vertical Cavity Surface Emitting Laser. Asian Journal of Chemistry, 24, 3194-3196.

[29] Marjani, S. (2013) Various Elements of Heat Sources within an Optimized Photonic Crystal Vertical Cavity Surface Emitting Laser: Influence of Hole Etching Depth. Asian Journal of Chemistry, 25, 4153-4156.

[30] Marjani, S. (2013) Optimization of an InGaAsP Vertical-Cavity Surface-Emitting Diode Lasers for High-Power Single-Mode Operation in 1550 nm Optical-Fibre Communication Systems. Asian Journal of Chemistry, 25, 4150-4152.

[31] Marjani, S., Faez, R. and Hosseini, S.E. (2013) Threshold Characteristics Analysis of InP-Based PhC VCSEL with Buried Tunnel Junction. The 21st Iranian Conference on Electrical Engineering (ICEE), Mashhad, 14-16 May 2013, 1-4. http://dx.doi.org/10.1109/iraniancee.2013.6599783

[32] Majdabadi, A., Marjani, S. and Sabaghi, M. (2014) Threshold Characteristics Enhancement of a Single Mode $1.55 \mu \mathrm{m}$ InGaAsP Photonic Crystal VCSEL for Optical Communication Systems. Optics and Photonics Journal, 4, 296-303. 
http://dx.doi.org/10.4236/opj.2014.410029

[33] Naeemi, M.A., Marjani, S. and Peiravi, A. (2014) Time to Failure Analysis of Single Mode Long-Wavelength InGaAsP Vertical-Cavity Surface-Emitting Lasers. The 22nd Iranian Conference on Electrical Engineering (ICEE), Tehran, 20-22 May 2014, 43-47. http://dx.doi.org/10.1109/iraniancee.2014.6999500

[34] Hajimiri, A. and Lee, T.H. (1999) Design Issues in CMOS Differential LC Oscillators. IEEE Journal of Solid-State Circuits, 34, 717-724. http://dx.doi.org/10.1109/4.760384

[35] Lee, S.-Y. and Hsieh, J.-Y. (2008) Analysis and Implementation of a 0.9-V Voltage-Controlled Oscillator with Low Phase Noise and Low Power Dissipation. IEEE Transactions on Circuits and Systems, 55, 624-627. http://dx.doi.org/10.1109/TCSII.2008.921574

[36] Razavi, B. (1996) A Study of Phase Noise in CMOS Oscillators. IEEE Journal of Solid-State Circuits, 31, $331-343$. http://dx.doi.org/10.1109/4.494195

[37] Wang, L., Upadhyaya, P., Sun, P., Zhang, Y., Heo, D., Chen, Y.-J. and Jeong, D. (2006) A 5.3GHz Low-Phase-Noise LC VCO with Harmonic Filtering Resistor. The IEEE International Symposium on Circuits and Systems (ISCAS), Island of Kos, 21-24 May 2006, 3237-3240.

[38] Hegazi, E., Sjoland, H. and Abidi, A. (2001) A Filtering Technique to Lower LC Oscillator Phase Noise. IEEE Journal of Solid-State Circuits, 36, 1921-1930. http://dx.doi.org/10.1109/4.972142

[39] Leeson, D.B. (1996) A Simple Model of Feedback Oscillator Noise Spectrum. Proceeding of IEEE, 54, 329-330. http://dx.doi.org/10.1109/PROC.1966.4682

[40] Rael, J. and Abidi, A. (2000) Physical Processes of Phase Noise in Differential LC Oscillators. The IEEE Custom Integrated Circuits Conference (CICC), Orlando, 21-24 May 2000, 569-572. http://dx.doi.org/10.1109/cicc.2000.852732

[41] Long, J., Foo, J.Y. and Weber, R.J. (2004) A 2.4 GHz Low Power Low Phase Noise CMOS LC VCO. IEEE Computer Society Annual Symposium on VLSI, Lafayette, 19-20 February 2004, 213-214.

[42] Chuang, Y.H., Lee, S.H., Yen, R.H. and Juang, M.H. (2006) A Low-Voltage Quadrature CMOS VCO Based on Voltage-Voltage Feedback Topology. IEEE Microwave and Wireless Components Letters, 16, 696-698. http://dx.doi.org/10.1109/LMWC.2006.885643 